\title{
Non-Linear Modal Analysis for Bladed Disks with Friction Contact Interfaces
}

\author{
Denis Laxalde ${ }^{* \dagger}$, Loï Salles ${ }^{\dagger \ddagger}$, Laurent Blanc ${ }^{\dagger}$ and Fabrice Thouverez ${ }^{\dagger}$
}

\begin{abstract}
A method for non-linear modal analysis of mechanical systems with contact and friction interfaces is proposed. It is based on a frequency domain formulation of the dynamical system's equations of motion. The dissipative aspects of these non-linearities result in complex eigensolutions and the modal parameters (natural frequency and modal damping) can be obtained without any assumptions on the external excitation. The generality of this approach makes it possible to address any kind of periodic regimes, in free and forced response. In particular, stability analysis in flutter applications can be performed.

Applications for the design of friction ring dampers for blisks and for the dynamical simulation of bladed disk with dovetail attachment are proposed. Finally, we propose a study of dynamical behaviour coupling with the calculation of fretting-wear at the interfaces based on non-linear modal characterization.
\end{abstract}

\section{Introduction}

Turbomachinery bladed disks often feature contact and friction interfaces. In traditional blade-disk assemblies, joints in blade attachments bring an important source of non-linear behaviour as well as some damping due to friction [1]. Also, friction damping is probably the most popular source dissipation in turbomachinery applications and the technologies used by manufacturers are numerous. These can be under-platform dampers for blade-disk assemblies or shrouds as in LP turbines for example [2]. Integrally bladed disks (or blisks) lack of connections and joints at which energy dissipation by friction could occur. As a consequence, these single-piece structures are

\footnotetext{
*Address all correspondence to this author.

†École Centrale de Lyon, Laboratoire de Tribologie et Dynamique des Systèmes, 36 avenue Guy de Collongue, 69134 Ecully Cedex, France

${ }^{\ddagger}$ Snecma - Safran group, 77550 Moissy-Cramayel, France
}

very weakly damped and can experience high levels of vibration leading to $H C F$ or failure risks. A major issue for these technologies is then to control their vibratory levels. A damping strategy for these structures has been proposed [3] which involves the use of friction ring dampers.

The modelling of contact and friction interfaces, independently from the technology, and their dynamical simulation is a complex issue which has to been undertaken for the design of modern turbomachinery components. It is a non-linear problem with several uncertainties. For the simulation of the non-linear dynamics, several approaches are described in the literature and the most popular ones are the harmonic balance based methods [4-9, 3]. Most of these methods are based on forced response calculations and this brings several limitations. First, the damping performances cannot be obtained straightforward with such methods. The distribution of forces and their level are often not exactly known and this yield a certain amount of uncertainties in the simulation process. Assumptions on the excitation forces then have to be made. Also, forced response might not always be the most critical issue and, in particular, flutter, among other aeroelastic stability issues, cannot be handled straightforward with such approaches.

Here, a new approach for the dynamical simulation of mechanical systems with friction contact interfaces is proposed. Based on a frequency domain formulation of the dynamical system's equations of motion, it aims at calculating the non-linear modal solutions. These solutions are denoted "complex non-linear modes" by analogy with the classical definition of complex modes in linear non-conservative systems. This approach features several advantages among which the possibility of a direct assessment of modal characteristics associated with the vibration amplitude is the most important. In particular, assumptions on the excitation characteristics are no longer needed. Also, forced response as well as flutter problems can be addressed. 
This approach appears particularly adapted to the study friction and contact interfaces. Several numerical applications are then proposed showing the relevance of this approach in industrial applications. In particular an investigation of the damping properties of a blisk using friction rings is presented.

A second example presents the dynamical simulation of a bladed disk with dovetail attachment, taking into account the friction damping at the interfaces. On this example, we also point out that friction at interfaces can produce wear. Fretting-wear, among other phenomena (buckling, fatigue, frettingfatigue,...), appends to be a significant cause of failure of blades and has became a major issue for turbomachinery manufacturers. Despite some technological answers were found to prevent these phenomena, wear at blade root cannot, in most situations, be avoided due to relative displacements and high contact strength. This phenomenon was studied experimentally [10] and numerically [11] for quasi-static situations, which corresponds to displacements of blade root due to centrifugal forces. However, these studies cannot explain all wear phenomena observed in turbomachinery. This motivates the present investigation which deals with the role of vibrations and dynamics in wearing blade roots. Qin et al. [12] have studied fundamental frequencies of turbine blades with geometry mismatch in fir-tree attachments, which can be caused by wearing out during service. They haven't explored coupling between vibration and wear at the contact interfaces. Wear is a very complex multiphysics phenomenon because hardness, plasticity, grain structure, temperature among others can change wear debris creation. We will simplify wear modelling using a discretised version of Archard's law [13]. Non-linear modal analysis coupled with fretting-wear seems to be a fairly adapted method to interpret easily results and moreover it appears to be faster than forced response calculation.

\section{Non-linear modal analysis}

The concept of non-linear modes has been initially proposed by Rosenberg [14] and then developed and extended by many authors [15-17]. As for nonautonomous systems, the periodic solutions of nonlinear autonomous systems can be studied using approximate methods. Here, a generalization of Harmonic Balance methods [18] is proposed with the view of dealing with free vibrations of dissipative au- tonomous dynamical systems. This class of methods have, among others, the advantage of being applicable to large size systems with strong non-linearities.

\subsection{The concept of non-linear complex modes}

By analogy with the complex modes of a linear system, a complex non-linear mode is defined as an oscillation of the autonomous system with (potentially) a phase difference between its degrees of freedom. This phase difference is the main distinction between complex and normal non-linear modes since the concept of unison does not appear anymore. Moreover, trajectories are no longer lines or curves normal to isoenergy curves but closed curves. Lastly, and still by analogy with linear complex modes, the eigenvalues of the characteristic equation are a priori complex and can be written as:

$$
\lambda=-\beta+i \omega
$$

with,

- $\omega=\omega_{0} \sqrt{1-\zeta^{2}}$, the damped natural angular frequency,

- $\omega_{0}$, the natural angular frequency,

- $\zeta=\beta / \omega_{0}$, the modal damping ratio.

To illustrate this, let us consider now the example of a strongly non-linear and dissipative system made of two oscillators and an elementary friction damper (as shown in figure 1).

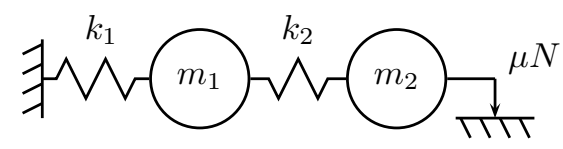

Figure 1. Oscillator with two degrees of freedom, including a friction damper

The equations of motion for this system are:

$$
\begin{gathered}
m_{1} \ddot{x}_{1}+c_{1} \dot{x}_{1}+k_{1} x_{1}+k_{2}\left(x_{1}-x_{2}\right)=0 \\
m_{2} \ddot{x}_{2}+c_{2} \dot{x}_{2}+k_{2}\left(x_{2}-x_{1}\right)+\mu N \tanh \frac{\dot{x}_{2}}{\epsilon}=0
\end{gathered}
$$

in which the restoring friction force is modelled by means of an hyperbolic tangent to approximate the sign function of the Coulomb friction model. In this 
model, $\mu$ and $N$ respectively refer to the coefficient of friction and normal load and $\epsilon$ controls the "slope" of the hyperbolic tangent; the smaller $\epsilon$ will be the larger the slope will be and the better the approximation of the sign function will be.

Figure 2 shows the trajectories of the system for fixed values of these friction parameters. These responses are calculated with the method described beneath. They represent the steady-state response for different vibration amplitudes and are independent of the initial conditions. At low levels, the trajec-

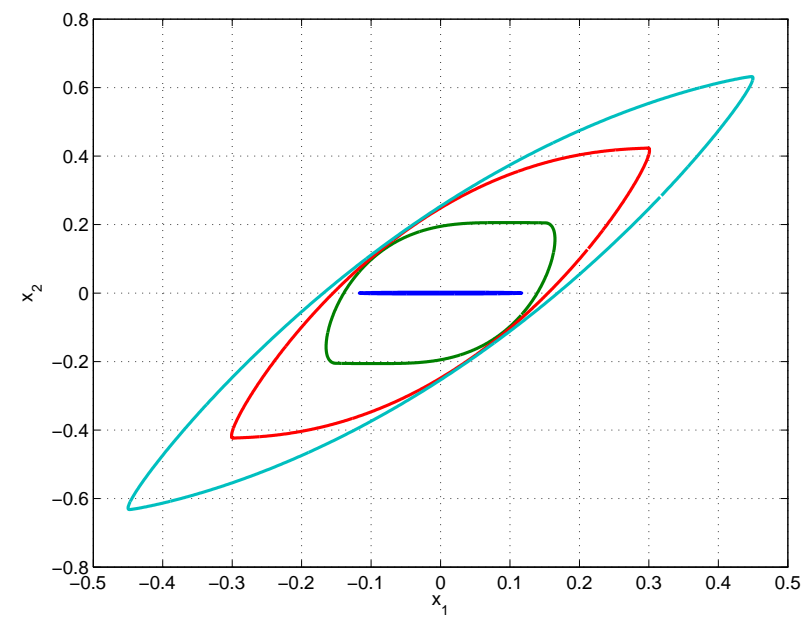

Figure 2. Trajectories of the complex non-linear modes for different amplitudes of vibration

tory looks like a straight line due to sticking contact at the mass $m_{2}$ (its equation is $x_{2}=0$ ) and the system is almost linear. When the energy level increases, non-linear effects appear. Their effect is, on the one hand, a curvature of the trajectories and, on the other hand, their "opening" (because of the non-linear dissipation), then a phase difference appears among degrees-of-freedom. This second effect cannot be taken into account through the analysis of the conservative system.

Following this definition of non-linear complex modes, we will now propose an associated formalism of the Harmonic Balance method in the frequency domain.

\subsection{Frequency-domain formulation of the complex eigenvalue problem}

Let us consider the discrete autonomous dynamical system described by the following second order dif- ferential equation:

$$
\mathbf{M} \ddot{\mathbf{x}}+\mathbf{C} \dot{\mathbf{x}}+\mathbf{K x}+\mathbf{f}(\mathbf{x}, \dot{\mathbf{x}})=0
$$

Besides conservative terms, this problem includes potentially dissipative terms represented by the linear damping and/or by the inner non-linear force $\mathbf{f}(\mathbf{x}, \dot{\mathbf{x}})$. The purpose is to calculate the modes of this nonlinear and non-conservative system. In terms of RitzGalerkin methods, we postulate the form of the solution of this free vibrations problem, that is the following Fourier series,

$\mathbf{x}(t)=\mathbf{Q}^{0}+\sum_{n=1}^{N_{h}} e^{-n \beta t}\left(\mathbf{Q}^{n, c} \cos n \omega t+\mathbf{Q}^{n, s} \sin n \omega t\right)$,

whose fundamental (angular) frequency, $\lambda=-\beta+i \omega$, is complex and represents the eigenvalue of the mode (see Eq. (1)).

The Fourier coefficients,

$$
\mathbf{Q}=\left[\mathbf{Q}^{0}, \mathbf{Q}^{1, c}, \ldots, \mathbf{Q}^{n, c}, \mathbf{Q}^{n, s}, \ldots, \mathbf{Q}^{N_{h}, s}\right]^{T}
$$

represent the eigenvector of the complex mode in the frequency domain.

The next step consists in formulating the algebraic complex eigenvalue problem associated with this definition according to equation (3). Velocities and accelerations are the derivatives of (4) and can be expressed through the following differential operator:

$$
\begin{aligned}
\nabla=\operatorname{diag}\left(0, \nabla_{1}, \ldots,\right. & \left.\nabla_{N_{h}}\right) \\
& \text { with } \nabla_{n}=n\left[\begin{array}{cc}
-\beta & \omega \\
-\omega & -\beta
\end{array}\right]
\end{aligned}
$$

which makes it possible to define the dynamic stiffness associated with the linear terms of equation (3) in the frequency domain:

$$
\begin{aligned}
\mathbf{Z}(\lambda)=\mathbf{K} \otimes \mathcal{I}_{2 N_{h}+1}+\mathbf{C} \otimes & \mathcal{I}_{2 N_{h}+1} \nabla \\
& +\mathbf{M} \otimes \mathcal{I}_{2 N_{h}+1} \nabla^{2}
\end{aligned}
$$

The non-linear terms, $\mathbf{f}(\mathbf{x}, \dot{\mathbf{x}})$, which depend on displacement and velocity are determined through an alternating frequency/time-domain (AFT) method (together with a Newton-like solver).

1. Given the vector $\mathbf{Q}$ of the frequency-domain coordinates of displacements and the eigenvalue $\lambda$, the expression of velocity in the frequency domain is $\nabla(\lambda) \mathbf{Q}$; 
2. These displacements and velocities are converted into the time domain through an inverse Fourier Transform:

$$
\tilde{\mathbf{x}}=\mathbf{Q}^{0}+\sum_{n=1}^{N_{h}}\left(\mathbf{Q}^{n, c} \cos n \omega t+\mathbf{Q}^{n, s} \sin n \omega t\right)
$$

3. The expression $\tilde{\mathbf{f}}(\mathbf{x}, \dot{\mathbf{x}})$ of the non-linear force in the time domain can be obtained either explicitly or by means of a time integration in the case of a system with contact interfaces;

4. The vector $\mathbf{F}$ of the frequency-domain coordinates of the non-linear force are given at last by a direct Fourier Transform:

$$
\begin{gathered}
\mathbf{F}^{0}=\frac{1}{T} \int_{-T / 2}^{T / 2} \tilde{\mathbf{f}}(\mathbf{x}, \dot{\mathbf{x}}) d t \\
\mathbf{F}^{n, c}=\frac{2}{T} \int_{-T / 2}^{T / 2} \tilde{\mathbf{f}}(\mathbf{x}, \dot{\mathbf{x}}) \cos n \omega t d t \\
\mathbf{F}^{n, s}=\frac{2}{T} \int_{-T / 2}^{T / 2} \tilde{\mathbf{f}}(\mathbf{x}, \dot{\mathbf{x}}) \sin n \omega t d t
\end{gathered}
$$

Let us remark the absence of logarithmic decrease $e^{-n \beta t}$ in the solution (4) for this $A F T$ formulation. Therefore, in the time domain step of the calculation of the frequency-domain components of the nonlinear forces, the displacements $\tilde{\mathbf{x}}$ and the velocities $\tilde{\mathbf{v}}$ as well as the non-linear forces $\tilde{\mathbf{f}}$ are periodic. To justify this assumption one could remember that this step of the calculation concerns only one period of the motion: at such a scale the maximum decrease of the amplitudes (evolving as $e^{-\beta T}$ ) remains generally weak. This also implies that the decrease of non-linear forces is the same as the decrease of other variables, this appears to be sane.

Finally, the eigenvalue equation is:

$$
\mathbf{Z}(\lambda) \mathbf{Q}+\mathbf{F}(\mathbf{Q}, \lambda)=0
$$

with the pairs $\{\mathbf{Q}, \lambda\}$ of eigenvectors and eigenvalues as unknowns.

\subsection{Modes normalization}

In order the complex eigen-problem (10) to be solved, one needs to define a normalization of the modes. Several approaches are possible such as a normalization with respect to the mass matrix or with respect to a chosen degree of freedom; an other solution, which is sometime used in the non-linear modes theory is to normalize the eigenmodes with respect to the energy.

In the present study, the modes are normalized with respect to a chosen degree of freedom. The amplitude of this degree of freedom thus defines the socalled modal amplitude:

$$
\mathbf{q}=\left[q^{c}, q^{s}\right]
$$

It has two components cosine and sine since the mode is complex. With this normalization, the eigenvectors can be expressed as:

$$
\mathbf{Q}^{c}=\boldsymbol{\Phi}^{c} q^{c} \text { and } \mathbf{Q}^{s}=\boldsymbol{\Phi}^{s} q^{s}
$$

The complex eigen-problem (10) can then be solved with a continuation process on the modal amplitude q.

\subsection{Application and validation of the proposed method}

In the following, the proposed method is illustrated using the example of the oscillator shown in figure 1 and mathematically described by equations (2). In a second step, a comparison with a direct timeintegration will be proposed to validate the method. All the frequency-domain analyses shown beneath have been computed with a sufficient number of harmonics. Because of the rather strong non-linearity, this number is quite high (between 10 and 20).

Figure 3 shows the evolution of the eigenfrequency and the modal damping in function of the level of displacement of mass $m_{1}$ for the first mode of the system. The asymptotic states with stuck friction damper $\left(x_{2}=0\right)$ and without a friction damper $\left(f_{N L}=0\right)$ are shown as dashed and dash-dotted lines. As the modal amplitude increases, the transition between stick and slip provokes a decrease of the eigenfrequency. In the intermediate slip-area (amplitude between 0.15 and 0.2 ), the modal damping reaches its maximum value. This last result is typical for friction systems whose maximal efficiency (in terms of damping) is reached in partial slip areas.

Figures 4 represents the phase planes for each degree of freedom and the hysteresis loops for different levels of modal amplitudes. For small vibration amplitudes the figures show a motion in the first degree of freedom and sticking behaviour in the degree of freedom that is associated to the friction damper. After an increase of the vibration amplitude the second degree of freedom comes into motion and the enclosed 


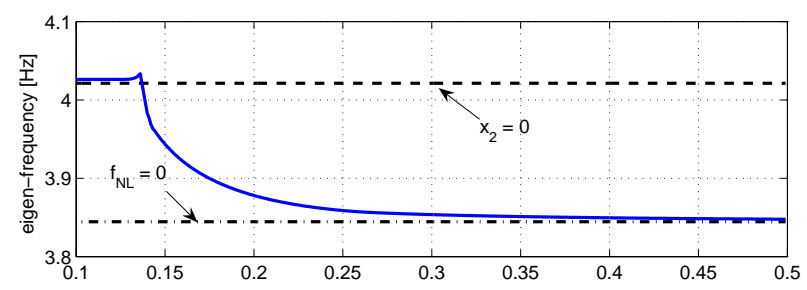

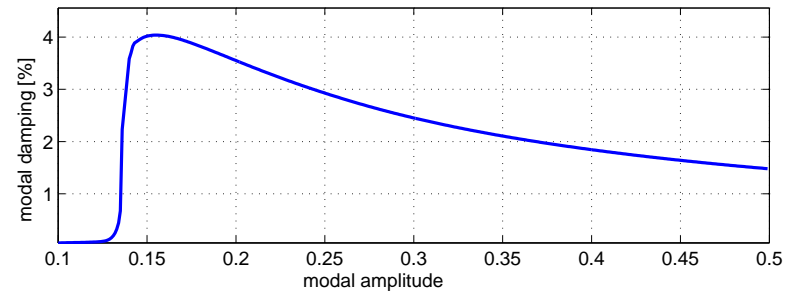

Figure 3. Eigenfrequency and modal damping as functions of the modal amplitude

hysteresis area increases. The diagrams show a clear non-linearity in the second degree of freedom.

\section{Analysis of the stability}

Next, lets us consider the effect of linear damping (of viscous-type here) on the non-linear modal analysis results. More specifically, when a negative destabilizing damping is introduced, the results of the proposed analysis make it possible to estimate the global stability of a mode. For example figures 5 show how the eigenfrequency and the modal damping evolve in the presence of a $2 \%$ negative damping. It is noticeable that for low or high modal amplitudes (corresponding to asymptotic configurations of slip, stick or total slip) the mode is unstable. Conversely, in the intermediate zone of modal amplitude (and partial slip), the modal damping is positive, which means that the mode is stable.

With the view on confirming these predictions of stability, a comparison has been made between the results of a direct integration of the free system under various initial displacement conditions and the results given by non-linear modal analysis in terms of limit cycles. This comparison validates the proposed method for the calculation of free responses.

Figure 6 gathers these results:

1. Low level initial condition: non-linear modal analysis predicts instability as well as direct integration does. Indeed, the vibratory level increases from the initial value and becomes stable on a limit cycle close to the one given by
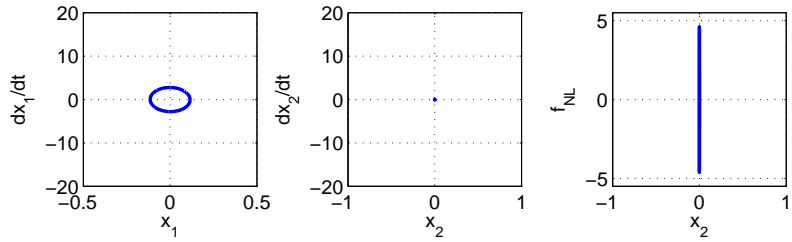

(a) Amplitude 0.115
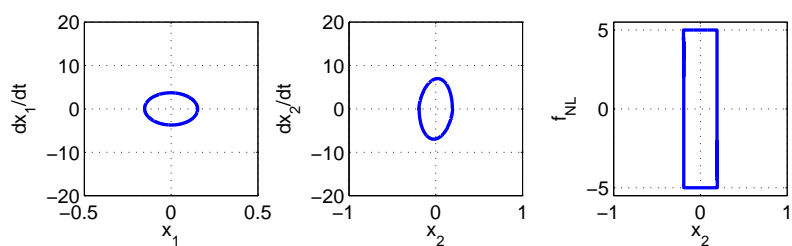

(b) Amplitude 0.150
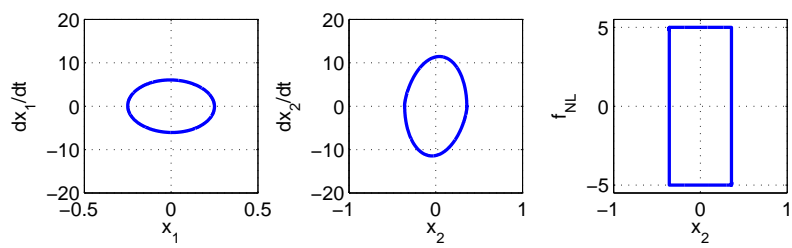

(c) Amplitude 0.247
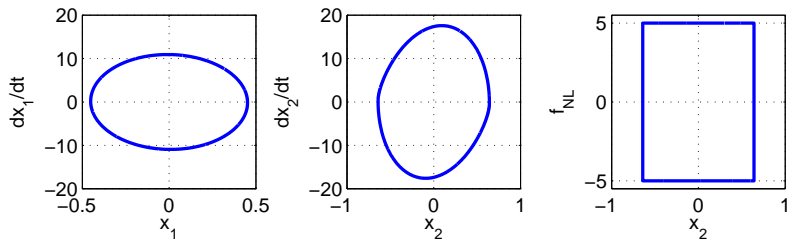

(d) Amplitude 0.449

Figure 4. Limit cycles for different modal amplitudes 

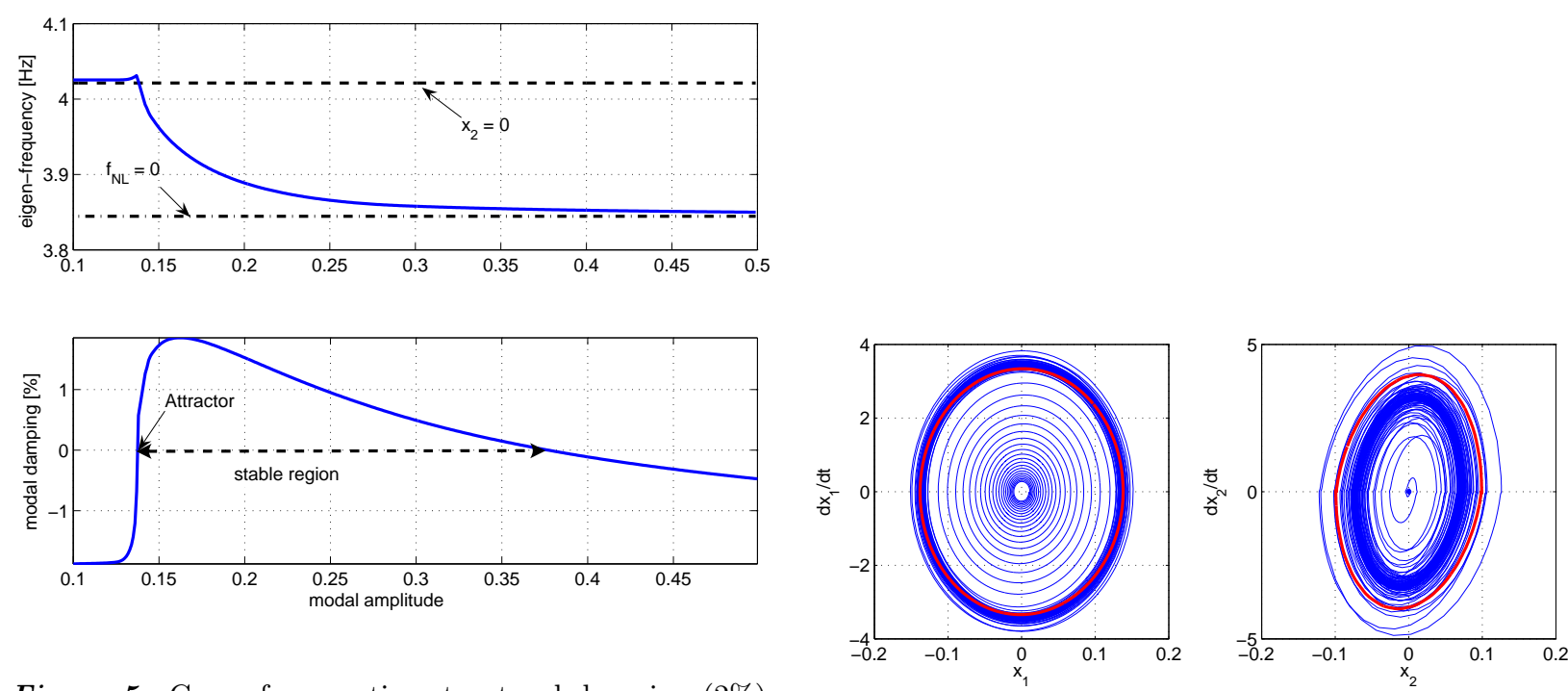

Figure 5. Case of a negative structural damping (2\%): eigenfrequency and modal damping as functions of the modal amplitude

the reference for a zero value of modal damping (attractor marks the point in figure 5).

2. Intermediate level initial condition: modal analysis predicts a stable solution. Indeed, modal damping is positive in this range of amplitudes and direct integration shows that the level decreases from the initial condition and becomes stable on the limit cycle given by the non-linear modes.

3. High level initial condition: non-linear modal analysis predicts no stable solution. This is confirmed by time-integration since the system diverges.

Remark: the stability prediction for a system with positive or negative linear damping can, in this example, be performed by the inclusion of damping $a$ posteriori. Indeed, modal damping evolves the same way with or without negative damping: damping produces only a shift of the modal damping curves toward negative values.

\section{Correlation between forced responses}

Lastly, a comparison is made between the results of non-linear modal analyses and frequency response functions for harmonic excitation of mass $m_{1}$. Frequency responses are calculated by the MultiHarmonic Balance method and results are validated by time-integration. Figure 7 gathers the frequency 


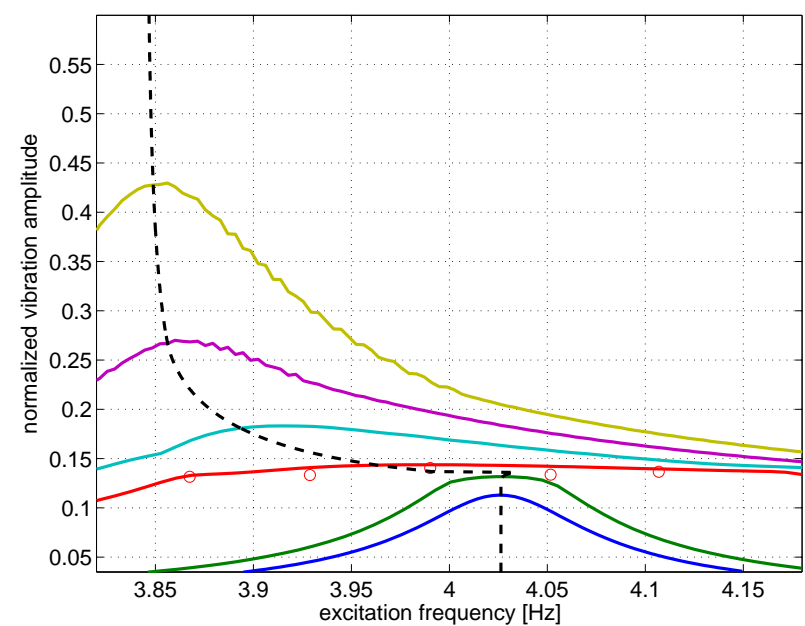

Figure 7. Correlation between frequency responses and non-linear modes: coloured solid lines, frequency responses for different levels of excitation; black dashed line, backbone curve predicted by the non-linear modes; $\circ$, stabilized points obtained by time-integration for a given level of excitation.

response results for different levels of excitation. The main conclusion is that the frequency / amplitude curve (backbone curve) obtained by non-linear modal analysis meets all the resonance peaks. This confirms the validity of the proposed method in the case of forced excitation.

\section{Application for the design of friction ring dampers for blisks}

The presented modal analysis method was applied to a single-piece bladed disk (blisk) of a HP compressor demonstrator with 36 blades fitted with a friction ring damper. The second bending mode, which mode shape is represented in figure 8 in form of a contour plot on the finite element model, is addressed.

The results of the non-linear modal analysis on this target mode are shown in figure 9. The behaviour is quite similar to that in the previous examples in terms of change of the natural frequency (figure 9a) and in the rate of modal damping (figure 9b) depending on the level of modal amplitude. For low vibration amplitudes, there is very little slip and the natural frequency remains constant while the modal damping rate is almost zero; which corresponds to the

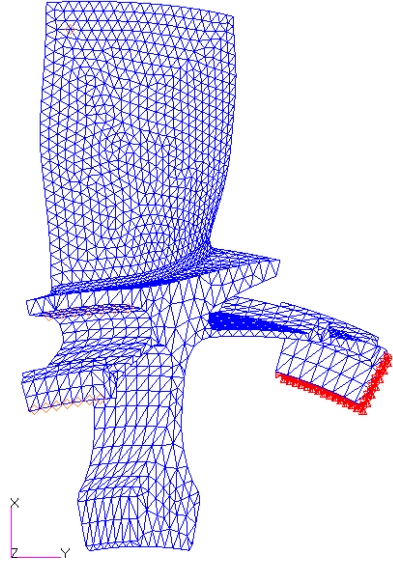

(a)

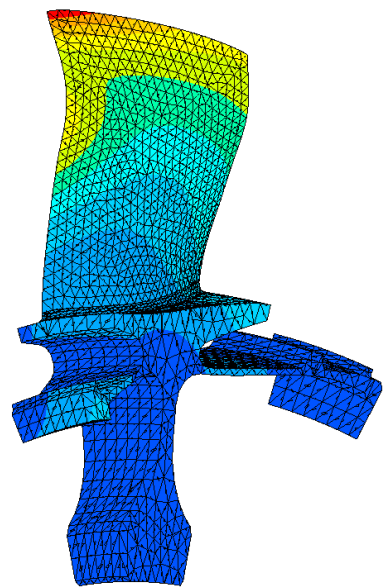

(b)
Figure 8. HP compressor blisk; (a) finite element mesh of a sector, (b) mode shape of the second bending mode at 4 nodal diameters.

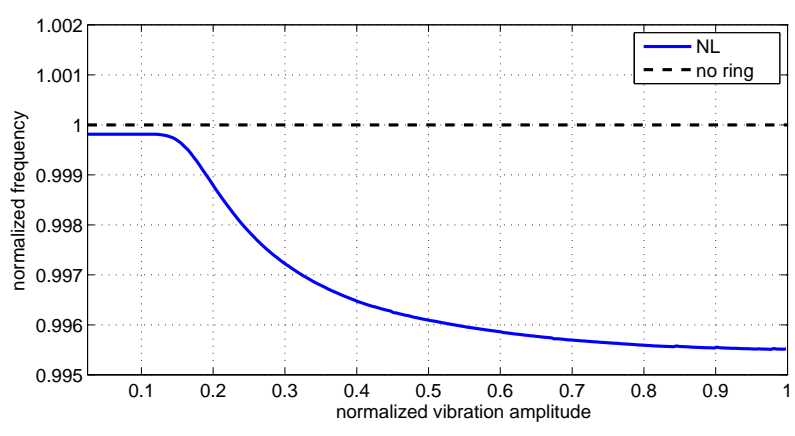

(a)

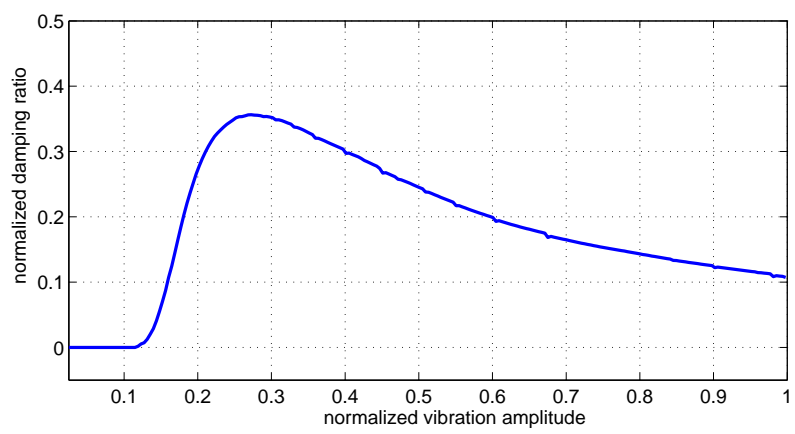

(b)

Figure 9. Results of non-linear modal analysis; change in the natural frequency (a) and in modal damping (b) depending on the level of vibration. 
asymptotic state for an adhering ring. In an intermediary amplitude range and from a certain threshold (amplitude of 0.4), the natural frequency diminishes abruptly while the damping rate increases to its maximum level (amplitude of 0.6); it is in this intermediary zone of sliding that friction damping is most effective. Lastly, it was observed that when the vibratory amplitude increases the damping rate gradually diminishes while the natural frequency increases once again with a trend towards an asymptotic state of a sliding ring.

One of the advantages of the non-linear modal analysis method proposed here is that the efficiency of a damping technology (such as friction rings) can be obtained nearly straightforward and whatsoever the excitation configuration or any other variable parameter.

As an example, we can study the sensitivity of friction ring damping with respect to the friction coefficient. On the same example, in figure 10 we show the change in modal data (natural frequency and modal damping) for several friction coefficient values. First, it can be seen that the lower the friction coefficient is, the lower the sliding threshold will be. Second, the difference between the limit values of this threshold is quite large (amplitude of 0.01 to 0.25 ), which shows that this type of friction damping device is highly sensitive to changes in the contact parameter. However, it can also be seen that maximum damping rate remains practically constant.

Such parametric studies, which are necessary in the design process of friction dampers, are quite easy to undertake and the results are also easy to interpret with the presented approach since the level of uncertainty is reduced to a minimum. In particular, the proposed modal approach makes it possible to evaluate the sensitivity of the damping properties with respect to any design (or environment) parameters without any assumptions on the excitation.

\section{Fretting-wear at blade-root interfaces}

This last section concerns a study of fretting-wear at blade-root interfaces. A calculation of wear is introduced in the non-linear modal analysis method; wear can then be considered as a new modal parameter. The case study is a compressor blade which $\mathrm{FE}$ mesh is shown in figure 11. This model is reduced to contact nodes of blade root $(2 \times 24$ nodes $)$

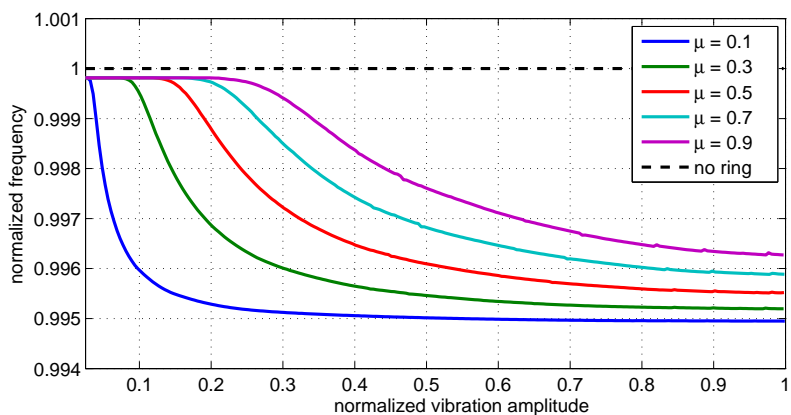

(a)

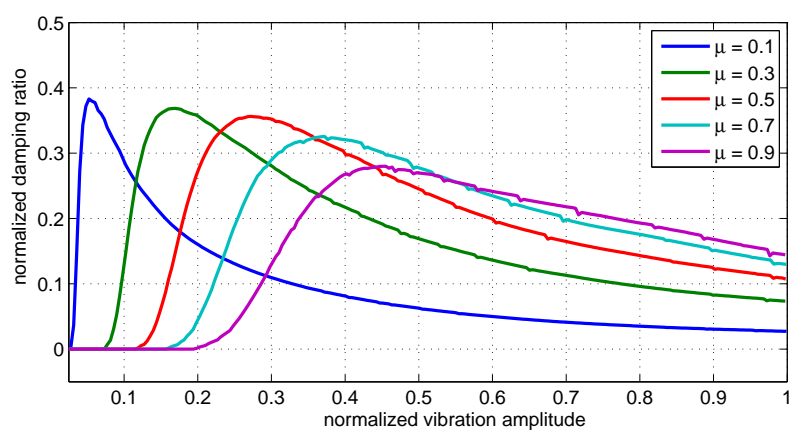

(b)

Figure 10. Non-linear modal analysis: influence of the friction coefficient; change in the natural frequency (a) and in modal damping (b) depending on the level of vibration.

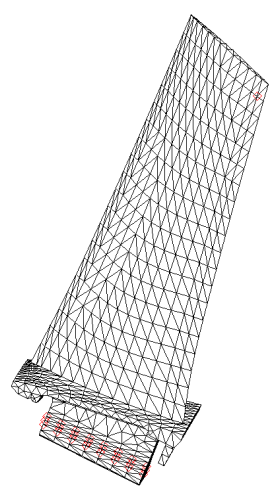

Figure 11. Finite element Model of blade with retained nodes 
and observation node at the trailing edge by means of a Craig \& Bampton method; 10 modal degrees-offreedom were also retained. The first bending mode is studied and the normal contact load due to centrifugal forces is approximated by an uniformly distributed force in each contact node.

In figure 12, the modal parameters (natural frequency and modal damping) without wear are represented in function of the modal amplitude. The behaviour is similar to previous examples (blisk with friction ring, or simple 2-DOF model) although the maximum damping appears to be higher than in the previous case.
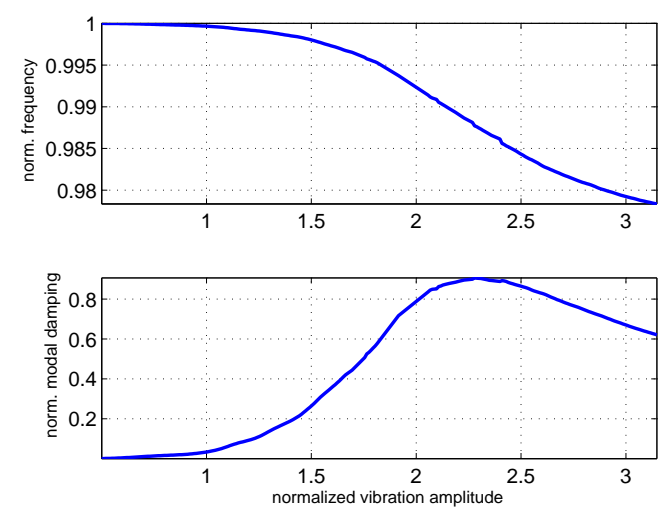

Figure 12. Result of non-linear modal analysis; change in the natural frequency and in modal damping depending on the level of vibration.

As mentioned in the introduction, we are now interested in studying the effects of fretting-wear of the contact interface which occur as relative displacements take place. Among other phenomena, frettingwear can be responsible for crack nucleation which can in turn produce blade failure. Here, fretting-wear is studied in the scope of dynamics and the Archard's law [13] is used to model this phenomenon. This law links wear volume to the product of the normal force and the sliding displacement. Archard's law is defined for volume that is why it must be adapted to finite element discretization. Thus, for each contact node $M$, we have,

$$
\dot{\mathbf{w}}^{M}=\frac{k_{w}}{I^{M}}\left|P_{N}^{M}\right|\left\|\dot{\mathbf{U}}_{T}^{M}\right\|
$$

where, $P_{N}^{M}$ is normal nodal force, $\dot{\mathbf{U}}_{T}^{M}$ is the vector of relative tangential velocity, $k_{w}$ is a wear constant and $I^{M}$ a weighting factor. The parameter $k_{w}$ must be de- terminated experimentally. McColl et al. [19] determine an averaged wear coefficient from the measured wear profile for a cylinder/flat contact. He integrates this averaged coefficient in the discretized Archard's law and simulates wear evolution in a "wear box" (FEM). In our case evaluation of averaged wear coefficient is performed for a punch/flat contact.

In general cases, wear changes material properties at the interfaces and their geometries. In this study, the change of Coulomb's coefficient of friction was neglected. It was then kept constant $(\mu=0.5$ corresponds to contact between blade and disk in titanium without hard-coating). Modification of geometry is taken into account using Stromberg's method [20]. In the penalty formulation of the contact problem with friction, wear was introduced as an initial gap on each period of the vibratory motion. This produces a reduction of the normal load at worn nodes. More details can be found in a subsequent paper [21].

The coupling of the modal analysis method with the calculation of wear is done assuming wear is a very slow phenomenon compared to non-linear vibration. Two time scales $(\tau, \eta)$ are introduced, respectively fast and slow and the coefficients of Fourier series (4) as well as modal parameters are functions of $\eta$. Equation (10) then becomes,

$$
\mathbf{Z}(\lambda(\eta)) \mathbf{Q}(\eta)+\mathbf{F}(\mathbf{Q}(\eta), \lambda(\eta), \eta)=0
$$

In this study, $\eta$ represents a multiply of numbers of fretting cycles. This new (slow) time scale leads to an additional dimension in modal analysis. Consequently, modal curves change into surfaces.

An example of modal parameters evolution with wear is shown in figure 13. It appears that damping increases with wear to a certain value and then decreases. Wear causes a decrease of the normal force, which allows interfaces nodes to be more often in slip situation. When the interface becomes too worn the dissipated energy decreases and damping decreases. Figures 14 show total nodal wear on blade-root surfaces. These figures show that surfaces don't wear in the same way. On one surface wear rate tends to zero when the number of fretting cycles increase. The second surface wears more and more as the vibration amplitude and fretting cycles increase. First, the evolution of wear follows modal damping curve of figure 12. To illustrate nodal wear, the wear evolution curve is drawn for an imposed vibration amplitude. Figure 15 shows different curves for different wear situations. There are nodes at which wear stops after certain number cycles, nodes which don't wear 


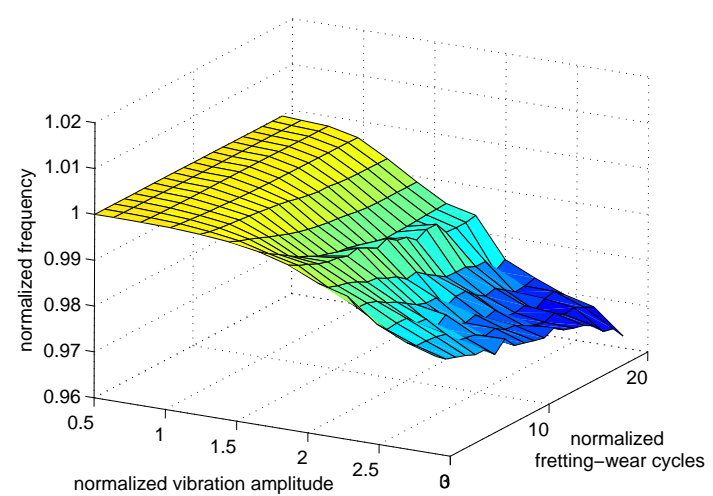

(a)

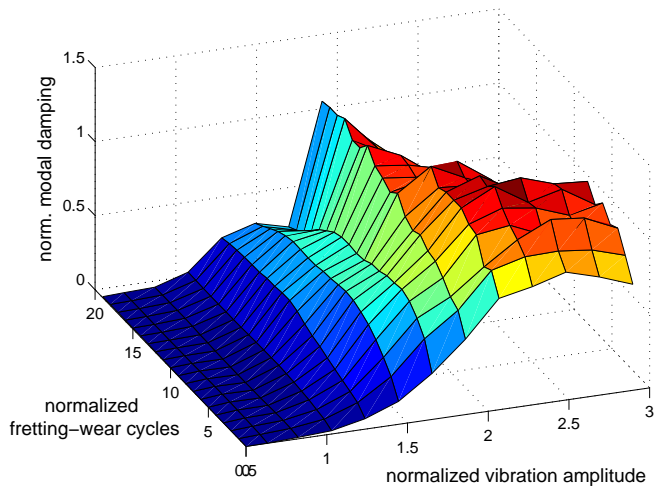

(b)

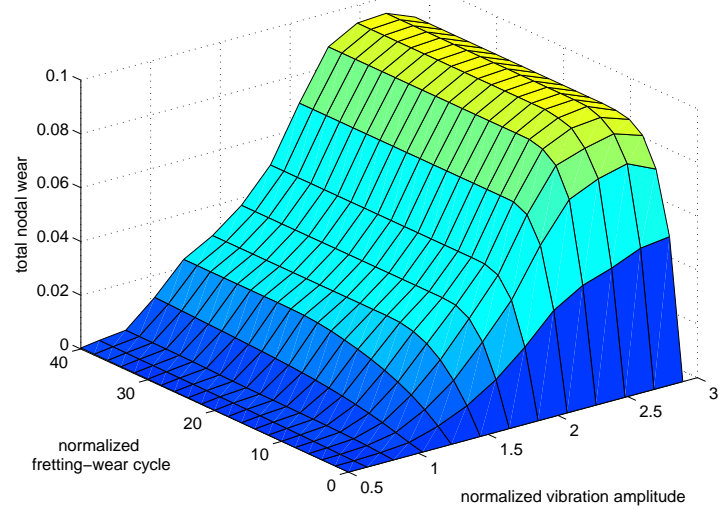

(a)

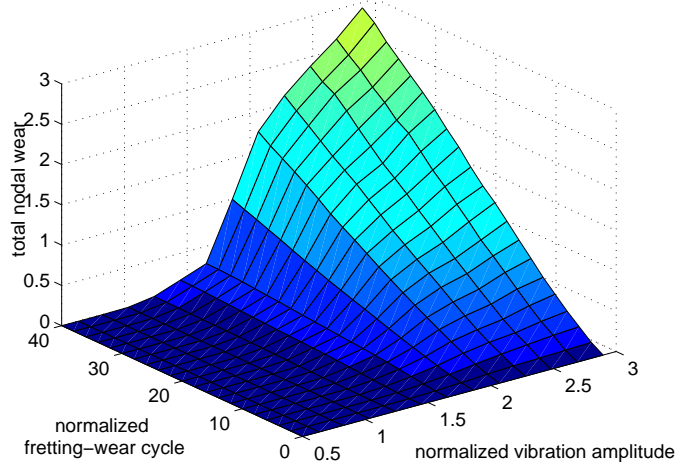

(b)
Figure 13. Result of non-linear modal analysis; change in the natural frequency (a) and in modal damping (b) depending on the level of vibration and fretting-wear cycle.
Figure 14. Result of non-linear modal analysis; Total nodal wear for one surface oof blade $\operatorname{root}(\mathrm{a})$ and for opposite surface (b) depending on the level of vibration and fretting-wear cycle. 


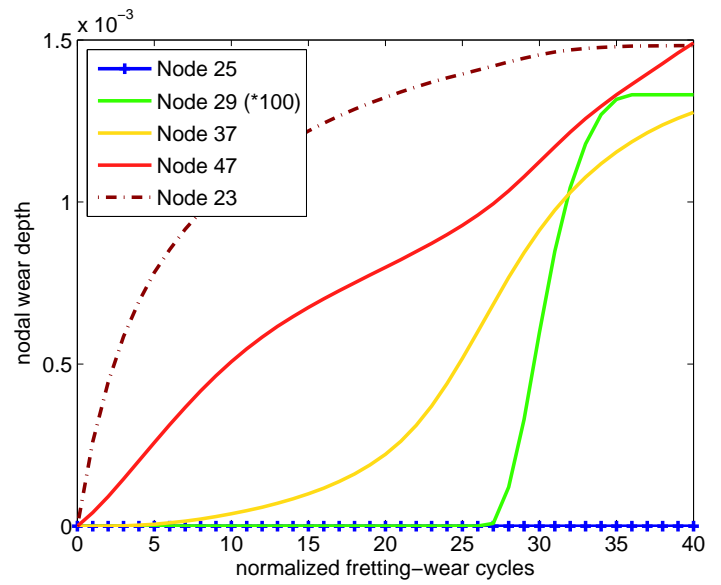

Figure 15. Nodal wear evolution at different contact node

and nodes which have complex wear kinematics. For an imposed vibration amplitude, the wear profile of each surface after 40 normalized cycles of modal wear is represented in figure 16 .

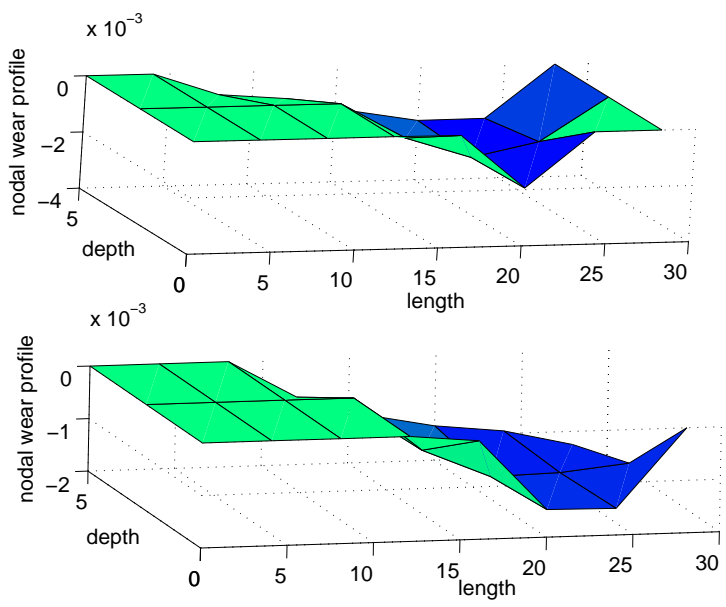

Figure 16. Nodal wear profile for each surface in contact

These calculations show coupling between wear and vibrations. In particular, wear rate isn't constant with the number of fretting cycles and can drastically changes when considering a larger number of fretting cycles. Thus, a single calculation of wear rate after one period of vibration isn't relevant to predict wear profile of blade roots. Also, it appears that modal damping may significantly change during blade's life.

This approach provides a valuable tool for the design and optimisation of blade-root profiles taking into account dynamical fretting-wear. The main point of using the modal approach, which makes no assumption on the excitation, in combination with the fretting-wear analysis is probably that it makes it possible to rank several designs of blade root with respect to their wear sensitivity.

\section{Conclusions}

A non-linear modal analysis method has been proposed; it is based on the concept of non-linear complex modes and on a frequency domain formulation of non-conservative autonomous dynamical problems. Thus, beyond frequencies and eigenmodes, this method supplies modal damping rates which, as other modal quantities, depend on the energy (or on the modal amplitude) of the system. This approach appears particularly relevant for the design of friction dampers as it has been shown on an industrial case-study involving a blisk equipped with a friction ring.

The second example shows a coupling of this nonlinear modal analysis method with wear calculation. Interesting results and information were obtained; in particular concerning the evolution of modal damping during turbomachinery's life. Calculation of modal wear is fast and gives useful information for bladeroot design.

\section{Acknowledgments}

Thanks go to Snecma for its technical and financial support. This work takes place in the framework of the MAIA mechanical research and technology program sponsored by CNRS, ONERA and SAFRAN Group.

\section{References}

[1] D. Charleux, C. Gibert, F. Thouverez, and J. Dupeux. Numerical and experimental study of friction damping in blade attachments of rotating bladed disks. International Journal of Rotating Machinery, 2006. doi:10.1155/IJRM/2006/71302. Article ID 71302,13 pages.

[2] J. H. Griffin. A review of friction damping of 
turbine blade vibration. International Journal of Turbo and Jet Engines, 7:297-307, 1990.

[3] D. Laxalde, F. Thouverez, and J.-P. Lombard. Forced response analysis of integrally bladed disks with friction ring dampers. Journal of Vibration and Acoustics, 132 (1):011013, 2010. doi:10.1115/1.4000763. oai:hal.archives-ouvertes.fr:hal-00453467.

[4] J. H. Wang and W. K. Chen. Investigation of the vibration of a blade with friction damper by hbm. Journal of Engineering for Gas Turbines and Power, 115:294-299, 1993. doi:10.1115/1.2906708.

[5] M. Berthillier, C. Dupont, R. Mondal, and J. J. Barrau. Blades forced response analysis with friction dampers. Journal of $\mathrm{Vi}$ bration and Acoustics, 120:468-474, 1998. doi:10.1115/1.2893853.

[6] A. A. Ferri and B. S. Heck. Vibration analysis of dry friction damped turbine blades using singular perturbation theory. Journal of Vibration and Acoustics, 120(2):588-595, 1998.

[7] J. Guillen and C. Pierre. An efficient, hybrid, frequency-time domain method for the dynamics of large-scale dry-friction damped structural systems. In IUTAM Symposium on Unilateral Multibody Contacts, Dordrecht, Netherlands, 1999.

[8] E. P. Petrov. Analytical formulation of friction interface elements for analysis of nonlinear multi-harmonic vibrations of bladed disks. Journal of Turbomachinery, 125(2):364, 2003. doi:10.1115/1.1539868.

[9] S. Nacivet, C. Pierre, F. Thouverez, and L. Jézéquel. A dynamic lagrangian frequency-time method for the vibration of dry-friction-damped systems. Journal of Sound and Vibration, 265:201-219, 2003. doi:10.1016/S0022-460X(02)01447-5.

[10] C. Paulin, S. Fouvry, and S. Deyber. Wear kinetics of ti $-6 \mathrm{al}-4 \mathrm{v}$ under constant and variable fretting sliding conditions. Wear, 259(1-6):292299, 2005. doi:10.1016/j.wear.2005.01.034.

[11] L. Gallego, D. Nélias, and C. Jacq. A comprehensive method to predict wear and to define the optimum geometry of fretting sur- faces. Journal of Tribology, 128(3):476, 2006. doi:10.1115/1.2194917.

[12] F. Qin, L. Chen, Y. Li, and X. Zhang. Fundamental frequencies of turbine blades with geometry mismatch in fir-tree attachments. Journal of Turbomachinery, 128(3):512, 2006. doi:10.1115/1.2187523.

[13] J. F. Archard. Contact and rubbing of flat surface. Journal of Applied Physics, 24(8):981-988, 1953. doi:10.1063/1.1721448.

[14] R. M. Rosenberg. The normal modes of nonlinear n-degrees-of-freedom systems. Journal of applied Mechanics, 30:595-611, 1962.

[15] W. Szemplinska-Stupnicka. "Non-linear normal modes" and the generalized Ritz method in the problems of vibrations of non-linear elastic continuous systems. International Journal of Non-Linear Mechanics, 18(2):149-165, 1983. doi:10.1016/0020-7462(83)90042-2.

[16] S. W. Shaw and C. Pierre. Normal modes for non-linear vibratory systems. Journal of Sound and Vibration, 164(1):85-124, 1993. doi:10.1006/jsvi.1993.1198.

[17] A. F. Vakakis, L. I. Manevitch, Y. V. Mikhlin, V. N. Pilipchuk, and A. A. Zevin. Normal Modes and Localization in Nonlinear Systems. Wiley series in nonlinear science, 1996. doi:10.1002/9783527617869.

[18] W. Szemplinska-Stupnicka. The behaviour of nonlinear vibrating systems, Vols $I$ and II. Kluwer Academic Publishers, 1990.

[19] I. R. McColl, J. Ding, and S. B. Leen. Finite element simulation and experimental validation of fretting wear. Wear, 256(11-12):1114-1127, 2004. doi:10.1016/j.wear.2003.07.001.

[20] N. Stromberg. An augmented lagrangian method for fretting problems. European journal of mechanics. A. Solids, 16(4):573-593, 1997.

[21] L. Salles, L. Blanc, F. Thouverez, and A. Gouskov. Analysis of a bladed disk with friction and fretting-wear in blade attachments. In Proceedings of ASME Turbo Expo, number GT2008-51112, Berlin, Germany, 2008. 